\title{
Advances in Treatment of Postpartum Rectus Abdominis Separation
}

\author{
Xiaohua Nong ${ }^{1, \dagger}$, Lihua Qin ${ }^{2, \dagger}$, Guosheng Su${ }^{3, *}$, Lijin Qin ${ }^{1, *}$, Han Wei ${ }^{1}$, Cailin Xu ${ }^{1}$, \\ Chunhong Pan ${ }^{1}$, Yuhua $\mathrm{Wu}^{1}$, Yulan Wei ${ }^{1}$ \\ ${ }^{1}$ Departmnt of Obstetrics and Gynecology, Baise People's Hospital, Southwest Hospital Affiliated to Youjiang Medical College for \\ Nationalities, Baise, China \\ ${ }^{2}$ Department of Osteoarthritis, Guigang Hospital of Integrated Traditional Chinese and Western Medicine, Guigang Red Cross Hospital, \\ Guigang, China \\ ${ }^{3}$ Department of Clinical Laboratory, Guigang Hospital of Integrated Traditional Chinese and Western Medicine, Guigang Red Cross Hospital, \\ Guigang, China
}

\author{
Email address: \\ 349679983@qq.com (Xiaohua Nong),787209349@qq.com (Lihua Qin),563449581@qq.com (Guosheng Su), \\ 1957612427@qq.com (Lijin Qin),8858381@qq.com (Han Wei),390386818@qq.com (Cailin Xu), \\ 13877682019@163.com (Chunhong Pan),158191524@qq.com (Yuhua Wu),273388177@qq.com (Yulan Wei) \\ * Corresponding author \\ $\dagger$ Xiaohua Nong and Lihua Qin are co-first authors
}

\section{To cite this article:}

Xiaohua Nong, Lihua Qin, Guosheng Su, Lijin Qin, Han Wei, Cailin Xu, Chunhong Pan, Yuhua Wu, Yulan Wei. Advances in Treatment of Postpartum Rectus Abdominis Separation. American Journal of Nursing Science. Vol. 7, No. 5, 2018, pp. 178-181.

doi: 10.11648/j.ajns.20180705.14

Received: July 30, 2018; Accepted: September 12, 2018; Published: October 9, 2018

\begin{abstract}
Objective: The study aims to explore the value of bionic electrical stimulation as a new clinical therapeutic instrument in the repair of postpartum rectus abdominis separation. Methods: The related literatures at home and abroad were searched and collected, and the research progress of clinical treatment of postpartum rectus abdominis separation was summaried, for the purpose to provide reference value for clinical research of postpartum rectus abdominis separation. Results: There was no study on biomimetic electrical stimulation combined with warm moxibustion belt in the treatment of postpartum rectus abdominis separation in all related literatures. Conclusion: A further and better exploration on the method of bionic electrical stimulation combined with warm moxibustion waistband in the treatment of postpartum rectus abdominis separation. This study is expected to provide a more scientific method for obstetric treatment.
\end{abstract}

Keywords: Postpartum, Postpartum Rectus Abdominis Separation, Treatment

\section{Introduction}

Rectus abdominis is located in the pubic symphysis and the pubic spine to the sternal xiphoid and the $5 \sim 7$ rib cartilage between the muscle tissue, which can be touched on both sides of the median line of the anterior abdominal wall [1]. With the growing of the fetus in the mother's womb, fetal pressure on the lumbar spine of pregnant women will also further increase the expansion of the abdominal wall of uterus which will cause the anterior rectus abdominis raphe of pregnant women gradual relaxation, while the linea alba separates to two sides, and this process is the separation of the rectus abdominis, after delivery of the fetus, the abdomen of the puerpera will show more obvious separation of the rectus abdominis [2-3]. At present, the corrective treatment of rectus abdominis separation has two methods, surgical and non-surgical, among them, non-surgical treatment is more acceptable for patients. Non-surgical treatment includes self-training, aerobic exercise, pseudo-bioelectric stimulation [4], etc. Among them, the bionic electric stimulation therapy can make the abdominal muscles of puerperas get all-round exercise to improve the treatment compliance, which has a good application prospect in the separation of postpartum abdominal rectus. 


\section{The Summary of Postpartum Rectus Abdominis Separation}

The levels of hormones in women during pregnancy will be a certain degree of change in order to facilitate the healthy development of fetus [5-6]. With the growth of fetus in the mother's body, the abdominal organs of pregnant women shift gradually to increase mechanical pressure on the abdominal wall, which leads to the stretching of the linea alba, continuous relaxation of rectus abdominis muscle, spacing increasing, resulting in the rectus abdominis separation. Due to lack of support force of the rectus abdominis, the intra-abdominal organs of patient continue to sag to compress the bladder, but also easy to cause leakage of urine [7]. Keshwani [8] put forward, the weakening of abdominal muscles strength will increase the compensatory overload work of other muscles, which will lead to the weakness of pelvic floor tissue and even severe back pain etc. The separation of the rectus abdominis after delivery not only leads to the state of the body fat, flabby skin, difficult to restore graceful figure, but also greatly affects normal life quality of puerperants, causing the puerperants to get the adverse reactions of backaches [9-10]. Therefore, it is the key to improve the quality of life to actively explore effective treatment of postpartum abdominal rectus separation.

\section{Application of Self- Training in the Postpartum Rectus Abdominis Separation}

According to the view of modern medicine, most women can start training from the third day after delivery (Cesarean section depends on the recovery of the wound). Methods as follows: patients are required to perform rehabilitation exercises at home every day, standing posture with contract abdomen, kneeling position with contract abdomen, kneeling position with stretch legs, leg-raising with supine position, driving leg with supine position, flat support and other rehabilitation actions, 10 consecutive times for a group to complete 3-4 groups a day.

\section{Application of Aerobic Exercise in the Postpartum Rectus Abdominis Separation}

Aerobic exercise refers to the exercise of the body with adequate oxygen supply, which including swimming, jogging, cycling, etc. When do the aerobic exercise, muscle contraction for a long time, heart and lung supplying oxygen to muscle, while transporting the waste from muscle, thereby repairing the muscle strain. Therefore, aerobic exercise has a certain repair effect on the rectus abdominis separation.

\section{The Application of Pseudo-Bioelectric Stimulation in the Postpartum Rectus Abdominis Separation}

The bionic electric stimulation mainly through the continuous stimulation of maternal abdomen to get contraction, and thus strengthen the intensive training of local muscles of puerperant's loose abdominal wall, so that the muscles gradually return to normal function. In the early stage of the use of pseudo-bioelectric stimulation therapy, low-frequency electrotherapy should be used to minimize the strong stimulation of current intensity on the maternal abdominal wall, generally without maternal pain for the appropriate limit, each treatment time can be controlled at $30 \mathrm{~min}$ or so, with the continuous adaptation of patient on the treatment effect of pseudo-bioelectric stimulation, the current stimulation can be strengthened gradually, but should pay attention to the maternal tolerance [11-14]. In order to further improve the repair effect of rectus abdominis muscle separation, the manual massage and self-exercise training etc can also be adopted at the same time for adjuvant therapy to strengthen the repair of the maternal rectus abdominis separation. Weiying Zhang [15] selected 44 cases of postpartum rectus abdominis separation as the research object, of which 23 patients received the pseudo-bioelectric stimulation therapy, the other 21 patients took self-training treatment, after statistical analysis, 10 days after receiving treatment of pseudo-bioelectric stimulation in patients with rectus abdominis separation was obviously better than that of accepted self-training. The result suggests that the clinical efficacy of pseudo-bioelectric stimulation therapy for the treatment of rectus abdominis separation is eutherapeutic, can effectively promote the postpartum recovery and the treatment effect is far superior to a single self-training method. Shuzhen Meng [16] selected 100 cases of puerperants who were diagnosed rectus abdominis separation after post partum as the research objects, which were randomly divided into observation group and contrast group, and the contrast group adopted the French PHENIX pelvic floor rehabilitation apparatus to make the bionic excitatory electrical stimulation on rectus abdominis separation; then the observation group adopted the bionic excitatory electrical stimulation of French PHENIX pelvic floor rehabilitation apparatus while increasing the manual massage, the results showed that, the improved effect of rectus abdominis muscle separatio of patients who were cured by pelvic floor rehabilitation apparatus combined with massage therapy was significantly better than the contrast group, the result of the study shows that, the use of bionic excitatory electrical stimulation combined with massage therapy is simple and easy, and the clinical effect is significant, and it has a positive effect to improve the treatment of postpartum rectus abdominis separation. 


\section{The Application of Moxa-Moxibustion in the Postpartum Rectus Abdominis Separation}

Moxibustion is a traditional Chinese medicine health care treatment method, it has two thousand years of history, and has a certain curative effect for the treatment of postpartum rectus abdominis separation. The first routine disinfection, use the 2 cun acupuncture needle to insert quickly, then put the moxa stick on the top of needle handle and ignite it, and place paper pad under the acupuncture to prevent burns, pull out the needle when wait until the moxa stick burns out, one time/day, 4 times for one menstrual cycle of treatment, continuous treatment of 4 menstrual cycle [17-18]. Should pay attention to the nutritional balance of diet, promoting blood circulation, advocating warm while avoiding cold, and advocating hot tea, porridge of red dates, fresh vegetables, etc, which all play a complementary treatment effect in the postpartum rectus abdominis muscle separation [19].

\section{The Application of Warm Moxibustion Belt in the Postpartum Rectus Abdominis Separation}

From the perspective of traditional Chinese medicine, vitality damage after childbirth, the sudden loss of blood and body fluid, vessels of the whole body being deficient, and more blood stasis, so there is a parlance of "postpartum more empty, more stasis" [20]. Warm moxibustion belt therapy as a method of external treatment, it's the use of special medicine bag of moxibustion health wormwood belt which is put into the electric heating belt, wearing the belt on the rectus abdominis, applying the warm moxibustion principle of the belt to the postpartum recuperation, which can bring the effect of warming main and collateral channels, activating blood circulation to dissipate blood stasis, dispelling cold and removing dampness, replenishing qi-blood, can effectively alleviate the pain symptoms of postpartum rectus abdominis separation and promote the healing of rectus abdominis separation.

\section{The Surgical Treatment for Postpartum Rectus Abdominal Separation}

The method of surgical treatment is abdominal wall plasty, it is through surgery to restore the tension of abdominal muscle and skin to maintain the shape of the abdomen, the way of surgery is to re-suture and reset the split rectus abdominis, if necessary, through appropriate means to restore the tension of the waist muscles. Whether it is from the restoration of normal anatomic structure and physiological functions, or from the view of clinical effect, for the situation of aneuros abdominal wall, abdominal wall plastic surgery can be said to be an ideal, thorough surgery. It has been widely developed both at home and abroad [21].

\section{Prospect}

The abdominal wall is an important part of human tissue, which can maintain body shape, stabilize pelvis and support abdominal organs, the abdominal wall plays an extremely important role in human's normal work and life. Rectus abdominis separation is not only abdominal loose with ugly look, abdominal relaxation, support force reduction, abdominal strength decrease, but will also lead to the symptom of maternal pain in the waist, and the bearing ability of the spine increases gradually [22]. Therefore, it is an important step to find a reasonable treatment plan to improve the restorative effects of postpartum rectus abdominis separation. As a safe and effective treatment mode, pseudo-bioelectric stimulation has high application value in the treatment of postpartum abdominal rectus separation. In addition, the role of warm moxibustion belt therapy is through the expansion of local peripheral blood vessels, to improve microcirculation, increase blood flow, eliminate tissue edema, accelerate tissue metabolism, activate vascular vasomotion, accelerate the local and systemic circulation, to play the role of promoting blood circulation for removing blood stasis and dredging the channels.

In summary, self-training, aerobic exercise, pseudo-bioelectric stimulation, moxibustion, warm moxibustion belt, etc., which all can promote the early rehabilitation of postpartum abdominal rectus separation. The treatment method of surgery will be adopted when the conservative treatment is ineffective. The next step, we will seek a better treatment program, explore the use of pseudo-bioelectric stimulation with warm moxibustion belt treatment of postpartum rectus abdomse muscle separation in order to achieve the best results, to more quickly and safely promote the early rehabilitation of postpartum rectus abdominis separation of women

\section{Acknowledgements}

With the support of the hospital function department and the help of colleagues, so the project can be accomplished, here express the gratitude for their support and help. Wish them good health, work smoothly, all the best!

\section{Fund Project}

Baise City of Guangxi Scientific Research and Technology Development Project (NO.: Encyclopedia 20170502)

\section{References}

[1] Pascoal, A. G, Dionisio, S. Cordeiro, F. etal. Inter-rectus distance in postpartum women can be reduced by isometric contraction of the abdominal muscles: a preliminary case-control study [J]. Physiotherapy, 2014, 100 (4):344-348. 
[2] Sancho, M. F., Pascoal, A. G., Mota, P. et al. Abdominal exercises affect inter-rectus distance in postpartum women: a two-dimensional ultrasound study [J]. Physiotherapy, 2015, 101 (3):286-291.

[3] Kulhanek, J., Mestak, O.. Treatment of umbilical hernia and recti muscles diastasis without a periumbilical incision $[\mathrm{J}]$. Hernia: the journal of hernias and abdominal wall surgery, 2013 , 17 (4):527-530.

[4] Linli Yang, Xueqin Mao. Effect observation of pseudo-bioelectric stimulation therapy on rehabilitation after artificial abortion $[\mathrm{J}]$. Chinese Journal of Family Planning, 2015, 23 (6): 417-418.

[5] Keshwani, Nadia, Mathur, Sunita, McLean, Linda et al. Validity of Inter-rectus Distance Measurement in Postpartum Women Using Extended Field-of-View Ultrasound Imaging Techniques $[\mathrm{J}]$. The journal of orthopaedic and sports physical therapy, 2015, 45 (10):808-813.

[6] Xinli Shang. Nursing of postpartum body restoration on women [J]. Chinese Journal of Modern Nursing, 2012, 5 (11): 22-23.

[7] Ying Jiang, Qun Qiao. One case of ultrasound manifestation of rectus abdominis separation $[\mathrm{J}]$. Chinese Journal of Ultrasound in Medicine, 2008, 24 (12): 1086-1086.

[8] Mota, Patricia, Pascoal, Augusto Gil, Carita, Ana Isabel et al. The Immediate Effects on Inter-rectus Distance of Abdominal Crunch and Drawing-in Exercises During Pregnancy and the Postpartum Period [J]. The journal of orthopaedic and sports physical therapy, 2015, 45 (10):781-788.

[9] Barbosa, S., DeSá, R. A. M., CocaVelarde, L. G. et al. Diastasis of rectus abdominis in the immediate puerperium: Correlation between imaging diagnosis and clinical examination [J]. Archives of gynecology and obstetrics, 2013, 288 (2):299-303.

[10] Bihua Zhou, Danmei Liao, Linyi Wei, et al. 1462 cases of women pelvic floor screening of postpartum 6 to 8 weeks [J]. Chinese Journal of Reproductive Health, 2014, 25 (1): 50-51.

[11] Beibei Lin, Yue e Zu, Xing Fan, et al. Al. The efficacy analysis of pseudo-bioelectric stimulation combined with biofeedback in the treatment of postpartum sexual dysfunction in women [J]. Chinese Journal of Sexology, 2016, 25 (3): 62-64.

[12] Yongli Wang, ShuMing He, ChenShu Liu, et al. The therapeutic effect of pseudo-bioelectric stimulation on endometrial thinning [J]. China Medical Innovation, 2012, 09 (21): 138-139.
[13] Ye Xu, Jia Wang, Yi Meng. The effect analysis of electrical stimulation combined with biofeedback on the rehabilitation of postpartum pelvic floor muscle $[\mathrm{J}]$. Chinese Journal of Medical Guidance, 2014, 9 (17): 230-231.

[14] Hui Qian. Psychological nursing of biofeedback treatment on postpartum pelvic floor muscle [J]. Digest of the world's latest medical information: continuous electronic journals, 2016, 16 (56): 34-35.

[15] Weiying Zhang, Jing Song, Huan Li. The efficacy observation of pseudo-bioelectric stimulation treatment on postpartum abdominal rectus separation [J]. Journal of Qiqihar Medical College, 2016, 37 (12): 14-15.

[16] ShuZhen Meng, Yan Mo, SuMei Ban, et al. The effect observation of bionic excitatory electrical stimulation combined with manipulation massage on postpartum rectus abdominis separation [J]. Chinese Journal of Traditional Chinese Medicine, 2014, 7 (B12): 110-110.

[17] Yan Ren, Ling Zhao, Lanlan Liu, et al. The research progress of temperature characteristics of different moxibustion therapy [J]. Chinese Journal of Traditional Chinese Medicine, 2014, 32 (10): 2348-2350.

[18] Aijun Hua, Yaqin Tang. The effect observation of moxibustion therapy combined with joint functional exercise for the treatment of arthralgia syndrome [J]. Chinese Journal of Rural Medicine, 2012, 19 (11): 31-32.

[19] Yanxia Feng, Jie Zhang, Yue Zhang. Research progress for the therapy of postpartum pelvic floor rehabilitation [J]. China family planning and Department of gynecology. 2016, 8 (8):3-6.

[20] Zhiyong Wang, Shuchang Song, Lichun Wang. The Clinical study of acupuncture combined with biomimetic stimulation in the treatment of post - stroke dysphagia [J]. Chinese Journal of Integrative Medicine on Cardio / Cerebrovascular Disease [J]. 2014, 12 (8):3-6.

[21] Yanmei Wang. The reason, prevention and treatment of rectus abdominis separation in pregnant women [J]. Chinese and foreign medical research, 2011, 9 (2):923-924.

[22] Ruimin Wei, Xia Xiao, Liqing Lv. Study on the effect of electrical stimulation of pelvic floor neuromuscular combined with rehabilitation massage on promoting the treatment of postpartum rectus abdominis separation [J] Maternal and Children Health Care of China. 2016, 31 (21): 4361-4364. 